\title{
Efeitos adversos agudos e conforto da interface máscara de mergulho como estratégia de suporte ventilatório durante a ventilação não invasiva
}

\section{Acute adverse effects and comfort of the diving mask interface as ventilatory support strategy during noninvasive ventilation}

Andréa da Nóbrega Cirino Nogueira ${ }^{1,2}$. Bárbara Galdino de Sousa ${ }^{1,2}$. Janaina Guia Sinhorelli ${ }^{1,2}$. Renata dos Santos Vasconcelos ${ }^{1,2}$. Soraya Maria do Nascimento Rebouças Viana ${ }^{1,2}$. Beatriz Amorim Beltrão ${ }^{2}$. John Edney Santos $^{3}$. Arnaldo Aires Peixoto Júnior ${ }^{1,2}$. Renan Magalhães Montenegro Júnior ${ }^{1,2}$. José Glauco Lobo Filho ${ }^{1}$.

1 Universidade Federal do Ceará (UFC), Fortaleza, Ceará, Brasil. 2 Hospital Universitário Walter Cantídio (HUWC/UFC/ EBSERH), Fortaleza, Ceará, Brasil. 3 Fundação Universidade Regional de Blumenau, Florianópolis, Santa Catarina.

\section{RESUMO}

Objetivo: avaliar os efeitos adversos agudos e conforto da interface máscara de mergulho como estratégia de suporte ventilatório durante a ventilação não invasiva. Metodologia: Estudo clínico, experimental e descritivo, realizado no laboratório da Unidade de Pesquisas Clínicas, da Universidade Federal do Ceará, no período de junho e julho de 2020. Foram estudados 12 voluntários saudáveis. Utilizou-se um modelo de suporte ventilatório não invasivo de circuito único, acoplado a máscara de mergulho, cilindro de ar comprimido (fluxo de $15 \mathrm{~L} / \mathrm{min}$ ) e válvula de PEEP $\left(5 \mathrm{cmH}_{2} \mathrm{O}\right)$, durante o período de 60 minutos. As variáveis avaliadas foram: parâmetros fisiológicos (frequência cardíaca, frequência respiratória, pressão arterial e saturação periférica de oxigênio), gasometria venosa, escala visual analógica e efeitos adversos relacionados a interface. Resultados: A amostra foi composta por 12 mulheres com idade média $25,58 \pm 3,47$. Não foi observada diferença estatisticamente significante entre os dados gasométricos quando comparado os valores antes e após o uso da interface. O nível de desconforto respiratório foi pontuado como leve durante o uso da interface. Os efeitos adversos com maior grau de problema foram: pressão da máscara, desconforto torácico, peso da máscara e pressão no ouvido. Conclusão: A interface máscara de mergulho parece ser viável, não apresentando graves níveis de efeitos adversos que inviabilize a sua utilização, porém para ampliar a sua aplicabilidade clínica são necessárias implementações de melhorias.

Palavras-chave: Ventilação não invasiva. Máscaras. Insuficiência respiratória.

\section{ABSTRACT}

Objective: to evaluate the acute adverse effects and comfort of the diving mask interface as a ventilatory support strategy during non-invasive ventilation. Methodology: Clinical, experimental and descriptive study, carried out in the laboratory of the Clinical Research Unit, of the Federal University of Ceará, in the period of June and July 2020. Twelve healthy volunteers were studied. A single-limb circuit noninvasive ventilatory support model was used, coupled with a diving mask, compressed air cylinder (flow of $15 \mathrm{~L} / \mathrm{min})$ and PEEP valve $\left(5 \mathrm{cmH}_{2} \mathrm{O}\right)$, during the period of 60 minutes. The variables evaluated were: physiological parameters (heart rate, respiratory rate, blood pressure and peripheral oxygen saturation), venous blood gases, visual analog scale and adverse effects related to the interface. Results: The sample consisted of 12 women with an average age of $25.58 \pm 3.47$. There was no statistically significant difference between the gasometric data when comparing the values before and after using the interface. The level of respiratory distress was scored as mild during the use of the interface. The adverse effects with the greatest degree of problem were: mask pressure, chest discomfort, mask weight and pressure in the ear. Conclusion: The diving mask interface seems to be feasible, with no serious levels of adverse effects that would make its use unfeasible, however, to expand its clinical applicability, improvements are necessary.

Keywords: Noninvasive Ventilation. Masks. Respiratory insufficiency.

Autor correspondente: Renata dos Santos Vasconcelos, Rua Conselheiro Tristão, 600, Centro, Fortaleza, Ceará. CEP: 60050-101. Telefone: +55 85 98898-8510. E-mail: renatavasconcelos23@gmail.com

Conflito de interesses: Não há qualquer conflito de interesses por parte de qualquer um dos autores.

Recebido em: 18 Fev 2021; Revisado em: 10 Mar 2021; Aceito em: 20 Abr 2021. 


\section{INTRODUÇÃO}

O uso da ventilação não invasiva (VNI) consiste na aplicação de uma pressão positiva ao sistema respiratório, através de uma máscara ou de outras interfaces, e está associada a redução da necessidade de intubação, mortalidade e custos do tratamento, motivo pelo qual essa terapia vem se tornando cada vez mais frequente no tratamento de pacientes com insuficiência respiratória aguda ou crônica agudizada. ${ }^{1,2,3,4}$

Ainda que a VNI possa ser usada em um amplo espectro de situações clínicas associadas à insuficiência respiratória, a sua eficácia é variável. A taxa de falha da VNI varia entre $5-40 \%$ dos casos, e os casos de falha estão associados a maior taxa de mortalidade. ${ }^{5}$ Dentre os principais fatores que determinam o sucesso da VNI, a escolha da interface representa 50-100\% de todas as complicações, sendo um dos efeitos adversos mais frequentes. ${ }^{6,7,8}$ A seleção, o encaixe e o manuseio muitas vezes são desafiadores, pois $25-33 \%$ dos pacientes adequadamente selecionados para a VNI têm má adaptação principalmente devido a problemas relacionados à máscara. ${ }^{9,10,11}$

A máscara facial total (MTF), semelhante à máscara de mergulho, é uma interface alternativa criada para aumentar a tolerância dos pacientes. A MFT cobre todo o rosto, gerando menos dor em pontos de pressão e vazamentos, e apesar de causar maior sensação de claustrofobia, é a interface que apresenta melhor aceitação pelos pacientes. ${ }^{8,9}$ Entretanto, a MFT tem a desvantagem de ter um volume interno grande $(875 \mathrm{~mL}) .^{12}$

A máscara de mergulho pode ser utilizada com pressão positiva contínua nas vias aéreas (CPAP) artesanal, e para que haja correto funcionamento não pode haver vazamento no sistema, pois não haverá compensação da fuga dos gases por não utilizar um ventilador próprio para VNI. Nesta modalidade de VNI necessita-se de geradores de fluxos de ar comprimido e oxigênio, um misturador destes gases, um sistema de conexão às vias aéreas - interface - e um dispositivo para a geração de pressão expiratória final (PEEP). Dentre os principais benefícios fisiológicos desse método destacam-se: melhora da oxigenação, reexpansão de áreas atelectasiadas, redução do shunt intrapulmonar, aumento da superfície de troca gasosa e melhora da relação ventilação-perfusão. ${ }^{13,14,15}$

Nesse contexto, a máscara de mergulho foi utilizada em algumas situações de emergência, como por exemplo, no enfrentamento da primeira onda da pandemia de COVID-19, onde indústrias e institutos acadêmicos colaboraram para resolver os problemas de escassez de suprimentos médicos em todo o mundo, e os desafios na adaptação de máscaras de mergulho de rosto inteiro para aplicações de saúde, como protetores faciais e máscaras respiratórias de sistemas não invasivos foram relatados como contribuição. ${ }^{16}$

Diante disso, objetivou-se avaliar os efeitos adversos agudos e conforto da interface máscara de mergulho como estratégia de suporte ventilatório durante a ventilação não invasiva.

\section{MATERIAL E MÉTODOS}

Trata-se de um estudo clínico, experimental e descritivo, realizado no Laboratório da Unidade de Pesquisas Clínicas, dos Hospitais Universitários da Universidade Federal do Ceará (UFC), no período de junho e julho de 2020. O estudo foi submetido e aprovado pela Comissão Nacional de Ética em Pesquisa (CONEP) (CAAE: 35482620.2.0000.5045).

Os critérios de inclusão do estudo foram voluntários saudáveis (profissionais de saúde, fisioterapeutas, enfermeiros etc) com atuação profissional na condução e manejo de aparelhos de ventilação mecânica e nível de $\mathrm{pCO}_{2}<45 \mathrm{cmH}_{2} \mathrm{O}$ verificada na gasometria venosa coletada antes da adaptação da interface. E os critérios de exclusão foram voluntários portadores de lesões de pele na face ou alguma condição que inviabilizasse a adaptação da interface no rosto do voluntário.

\section{Protocolo do estudo}

Utilizou-se um modelo de suporte ventilatório não invasivo de circuito único, acoplado a: interface máscara de mergulho do tipo facial total (modelo de superfície CYCLOP, fabricante Seasub $^{\circledR}$ ), cilindro de ar comprimido com fluxo de $15 \mathrm{~L} /$ min (evitar a reinalação de $\mathrm{CO}_{2}$ ), válvula de PEEP ajustada em $5 \mathrm{cmH}_{2} \mathrm{O}$, filtros de ar antibacteriano e umidificador respiratório (HMEF - Heat and moisture Exchanger Filter, marca Covidien ${ }^{\circledR}$ ) entre o circuito-interface e interfaceválvula de PEEP, e manovacuômetro para monitorizar o nível de PEEP durante o experimento (Figura 1).

Figura 1. Voluntária adaptada à interface máscara de mergulho (seta preta) conectada ao cilindro de ar comprimido (seta vermelha) e válvula de PEEP (seta branca).

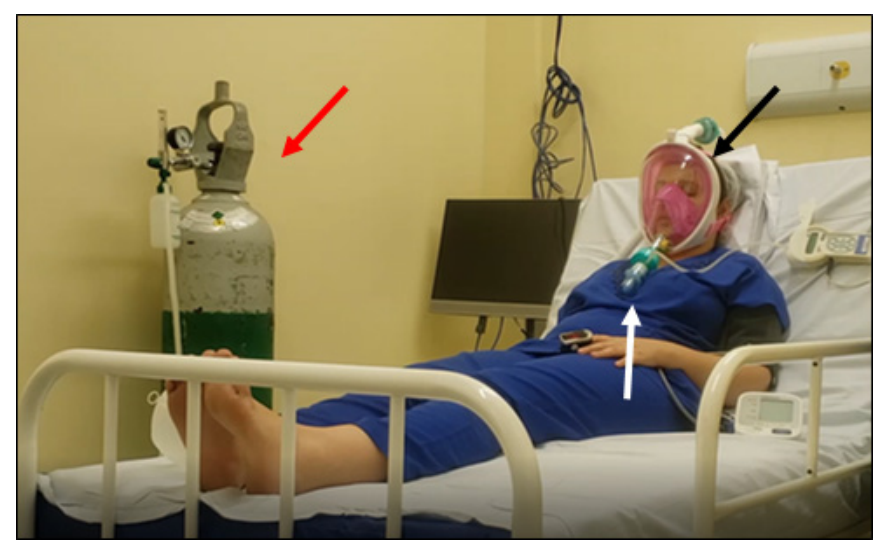

Os profissionais de saúde foram convidados a participar da pesquisa, obedecendo aos critérios de inclusão e exclusão. No dia dos testes, o voluntário saudável foi informado sobre as atividades do estudo e esclarecido suas dúvidas a respeito da utilização da máscara de mergulho, e sobre o instrumento de coleta de dados. Inicialmente foi avaliada a estabilidade clínica do voluntário e o participante foi orientado a retirar todos os 
adornos e prender o cabelo com uma touca descartável, em seguida foi posicionado em decúbito dorsal, cabeceira elevada $\left(30^{\circ}\right)$ para a mensuração dos sinais vitais e coleta da gasometria venosa, que foi realizada antes e ao final do uso da interface.

Após a montagem do sistema, foi realizada a adaptação da interface máscara de mergulho associada a CPAP, que foi colocada no voluntário por dois profissionais devidamente treinados, ${ }^{17}$ e conectada ao cilindro de ar comprimido com fluxo de $15 \mathrm{~L} / \mathrm{min}$, este foi mantido com o propósito de evitar a reinalação de dióxido de carbono $\left(\mathrm{CO}_{2}\right)$. O suporte ventilatório foi aplicado no tempo total de 60 minutos, e durante todo esse período utilizou-se um nível de pressão constante nas vias aéreas. Porém, caso o voluntário apresentasse algum sinal de instabilidade clínica durante o uso da interface como bradicardia ( $\mathrm{FC}<50 \mathrm{bpm}$ ) ou hipotensão arterial importante (PAM $<65 \mathrm{mmHg}$ ) o protocolo do estudo era interrompido.

Durante a realização do protocolo o voluntário foi monitorizado no repouso e a cada 15 (quinze) minutos quanto à: frequência cardíaca (FC), frequência respiratória (f), pressão arterial (PA), oximetria de pulso $\left(\mathrm{SpO}_{2}\right)$ e quanto ao nível de desconforto em uso da interface através de uma escala analógica visual EVA, essa escala pontua o nível de desconforto de 0 a 10 , onde 10 (dez) foi a nota atribuída para percepção de desconforto intenso quanto ao uso da interface e 0 (zero) quando a interface for muito confortável. E a gasometria venosa foi coletada no início e ao final do experimento.

Ao final do uso da máscara, foi aplicado um questionário padronizado de efeitos adversos da interface, incluindo dor em vários pontos de pressão da máscara, irritação nos olhos, percepção incômoda de vazamentos, sensação de ressecamento nasal e oral, dor de cabeça, dor na testa, dor na bochecha, dificuldade de respirar, barulho do equipamento e sensação de claustrofobia. Todos esses problemas foram pontuados de 0 a 3 pelo voluntário, sendo zero quando não foi considerado um problema e três quando foi considerado um grande problema.

Dessa forma, as variáveis do estudo foram: os parâmetros fisiológicos ( $\mathrm{FC}, \mathrm{f}, \mathrm{PA}, \mathrm{SpO}_{2}$ ), gasometria venosa, escala visual analógica e efeitos adversos relacionados à interface (Figura 2).

Figura 2. Protocolo do estudo.

\begin{tabular}{|c|c|c|c|c|}
\hline \multirow[t]{2}{*}{$\begin{array}{c}\text { Repouso } \\
\text { (FC, f, PA, } \\
\mathrm{SpO}_{2} \\
\text { Gasometria } \\
\text { venosa e EVA) }\end{array}$} & & $\begin{array}{c}30 \text { min } \\
\left(F C, f, P A, S^{\prime} O_{2}\right. \\
\text { e EVA })\end{array}$ & & $\begin{array}{c}60 \text { min } \\
\left(\mathrm{FC}, \mathrm{f}, \mathrm{PA}, \mathrm{SpO}_{2}\right. \\
\text { Gasometria } \\
\text { venosa, EVA e } \\
\text { efeitos adversos) }\end{array}$ \\
\hline & $\begin{array}{c}15 \text { min } \\
(F C, f, P A, \\
\mathrm{SpO}_{2} \text { e EVA) }\end{array}$ & & $\begin{array}{c}45 \text { min } \\
\text { (FC, f, PA, } \\
\mathrm{SpO}_{2} \text { e EVA) }\end{array}$ & \\
\hline
\end{tabular}

\section{Análise estatística}

Para a análise descritiva das variáveis gasométricas calcularamse medianas, valores máximos e mínimos de acordo com a distribuição da amostra, sendo utilizado o teste de Wilcoxon para confirmação da hipótese nula. Em virtude da classificação dos escores dos efeitos adversos ser decorrente de um achado subjetivo de cada voluntário, optou-se pela categorização dos mesmos, sendo essas variáveis categóricas descritas em frequências absolutas.

\section{RESULTADOS}

Foram selecionados para o estudo 22 voluntários saudáveis. Destes 22 voluntários, 8 foram excluídos por retenção de $\mathrm{CO}_{2}$ e 2 interromperam o protocolo por bradicardia durante o uso da interface, restando, portanto, 12 voluntários estudados (Figura 3).
A Tabela 1 mostra as características demográficas dos voluntários do estudo. A média de idade foi de 25,58 $\pm 3,47$, IMC de 26,6 44,27 e $100 \%$ da amostra foi composta por voluntários do sexo feminino.

Tabela 1. Características demográficas dos voluntários do estudo.

\begin{tabular}{lc}
\hline Idade (anos) , média \pm DP & $25,58 \pm 3,47$ \\
$\mathrm{IMC}\left(\mathrm{Kg} / \mathrm{m}^{2}\right)$, média $\pm \mathrm{DP}$ & $26,6 \pm 4,27$ \\
Gênero $(\%)$ & \\
Feminino & 100 \\
Masculino & 0 \\
Profissão (\%) & \\
Enfermagem & 66,6 \\
Fisioterapia & 33,4 \\
\hline
\end{tabular}

$\mathrm{IMC}=$ índice de massa corpórea, $\mathrm{DP}=$ desvio padrão. 
A Figura 4 mostra as variáveis de frequência cardíaca (FC), pressão arterial média, frequência respiratória (f) e saturação periférica de oxigênio $\left(\mathrm{SpO}_{2}\right)$, não sendo observada altos níveis de variação desses parâmetros durante o uso da interface.

A Figura 5 mostra as variáveis gasométricas dos voluntários do estudo. Não foi observada diferença estatisticamente significante entre os níveis de $\mathrm{pCO}_{2}(\mathrm{p}=0,928), \mathrm{pH}(\mathrm{p}=0,239)$ e $\mathrm{HCO}_{3}(\mathrm{p}=0,433)$ durante o uso da interface.

A Figura 6 mostra a variação da escala visual analógica (EVA) de desconforto da interface utilizada no estudo. Apesar dos voluntários terem referido leve desconforto durante todo o protocolo do estudo, notou-se um discreto aumento até 15 minutos de uso, com manutenção do nível de desconforto pontuado com leve até o final dos 60 minutos, não sendo observado nenhuma situação de desconforto moderado ou intenso durante o uso da interface.

A Figura 7 ilustra os efeitos adversos avaliados ao final do uso da interface. Os efeitos adversos com maior grau de problema relatados foram a pressão da máscara e o desconforto torácico $(\mathrm{n}=2 ; 16,67 \%)$; moderado, pressão da máscara e dor na testa $(n=3,25 \%)$; discreto, irritação na pele $(n=6 ; 50 \%)$, e nenhum voluntário relatou sensação de claustrofobia com uso da interface. Os voluntários referiram também efeitos diferentes dos categorizados no instrumento de coleta de dados, como desconforto relacionado ao peso da máscara e a pressão no ouvido ( $\mathrm{n}=2 ; 16,67 \%$ ) como grande problema, dificuldade de expirar e câimbra na região da mandíbula $(n=1 ; 8,33 \%)$.

Figura 3. Fluxograma da seleção dos pacientes do estudo.

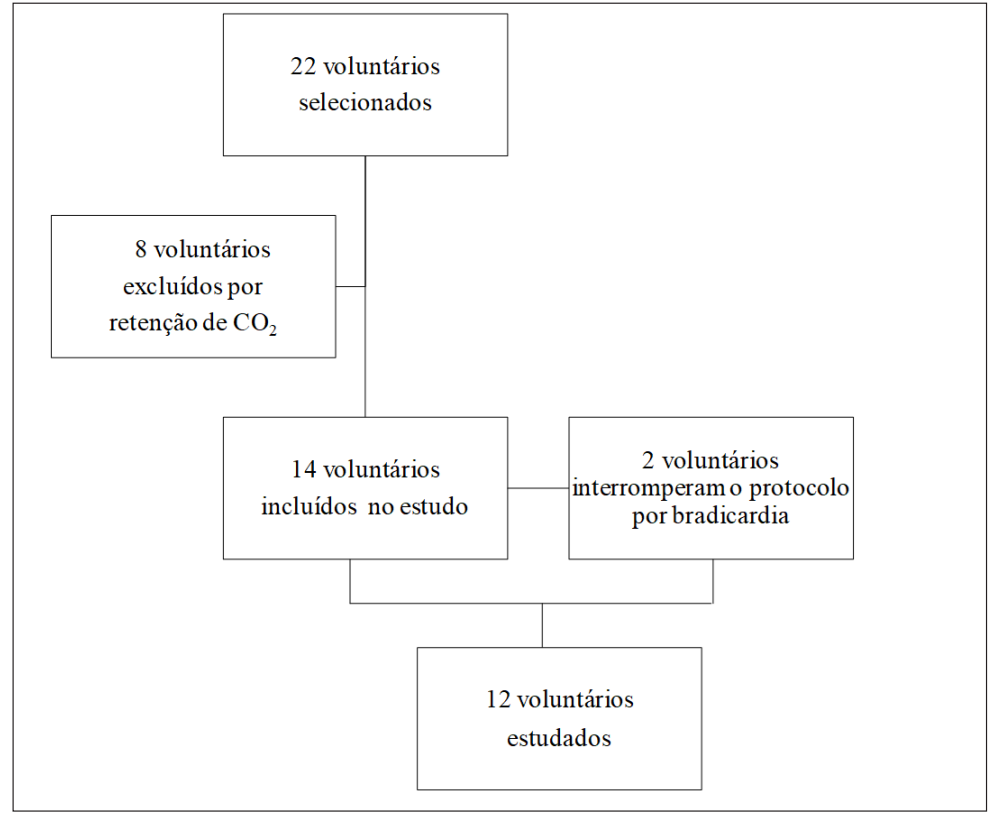

Figura 4. Parâmetros fisiológicos dos voluntários do estudo.

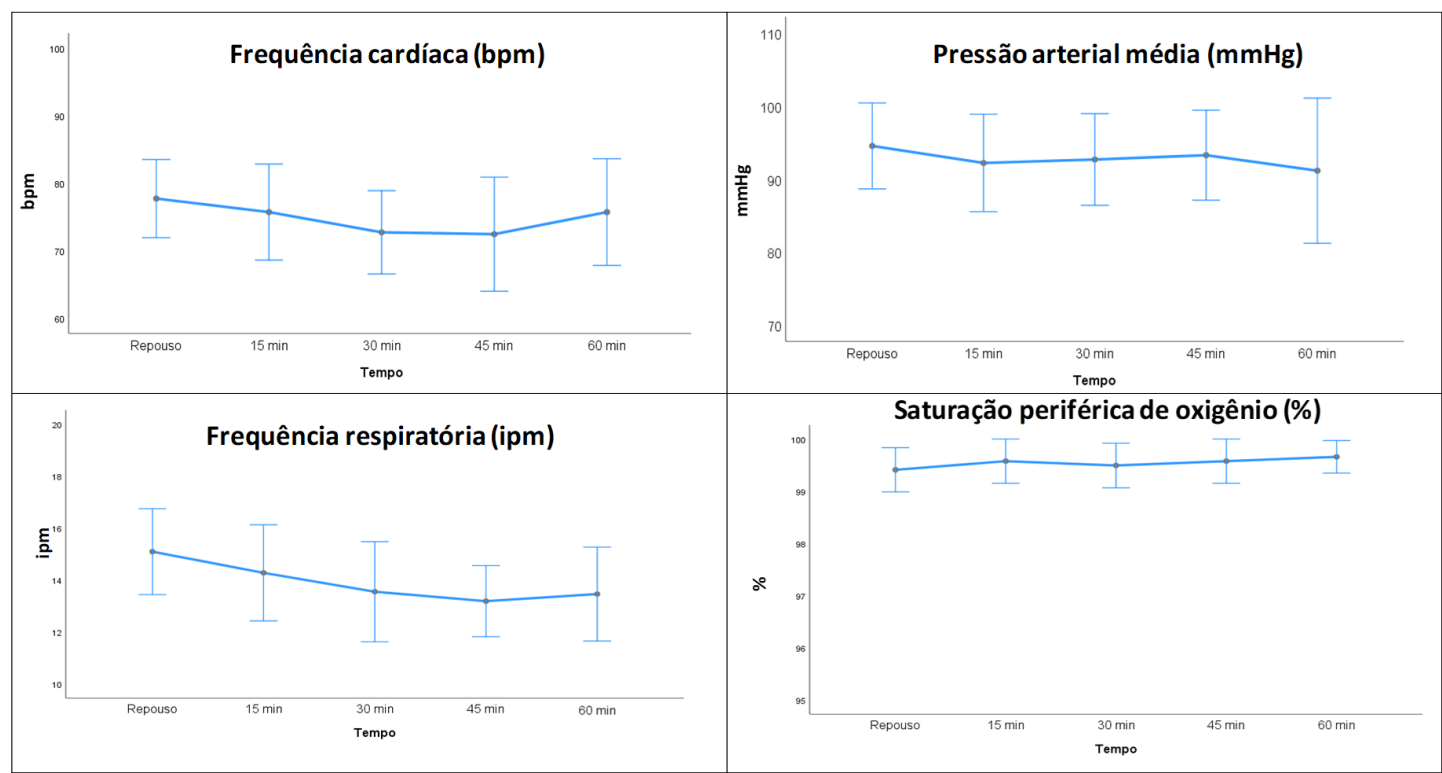


Figura 5. Variáveis gasométricas dos voluntários do estudo.

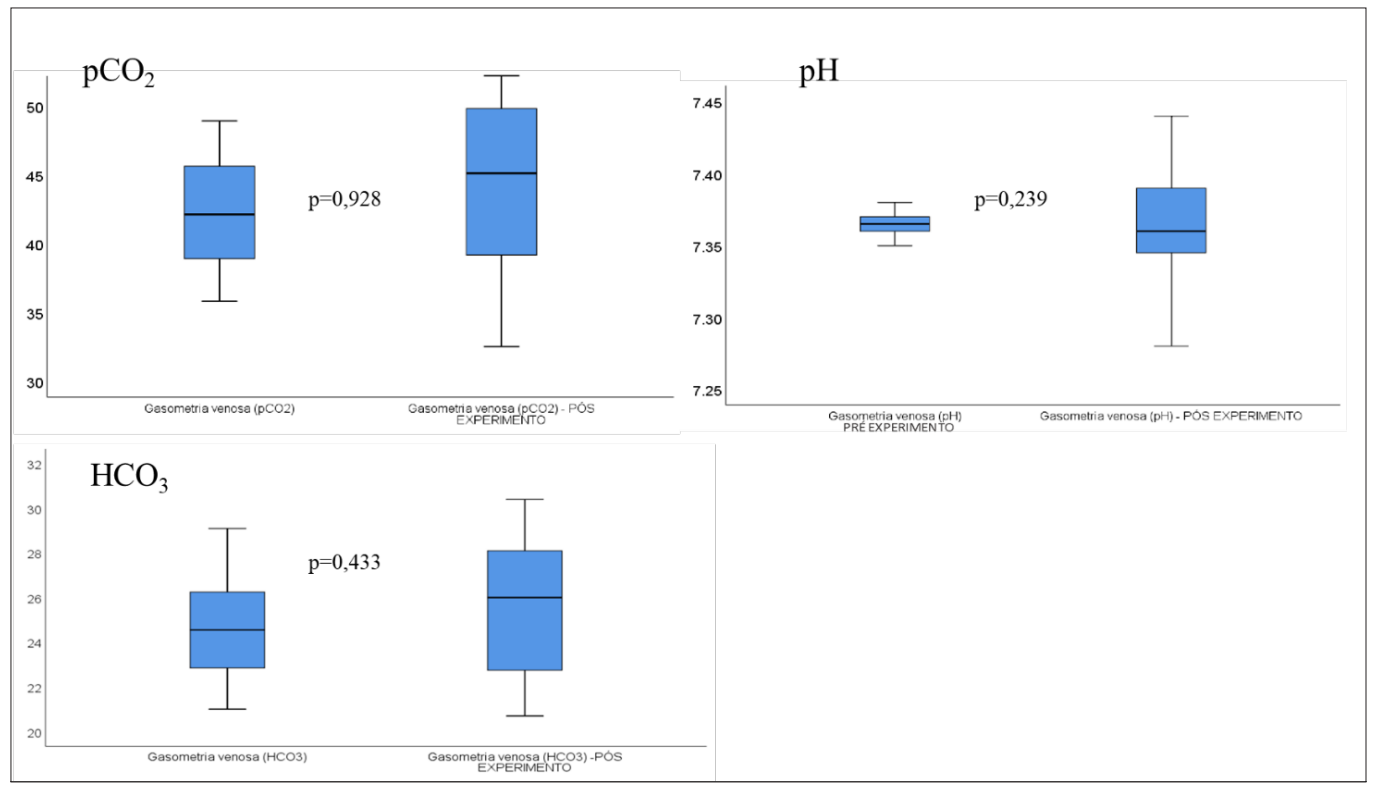

Figura 6. Variação da escala visual analógica (EVA) de desconforto da interface utilizada no estudo.

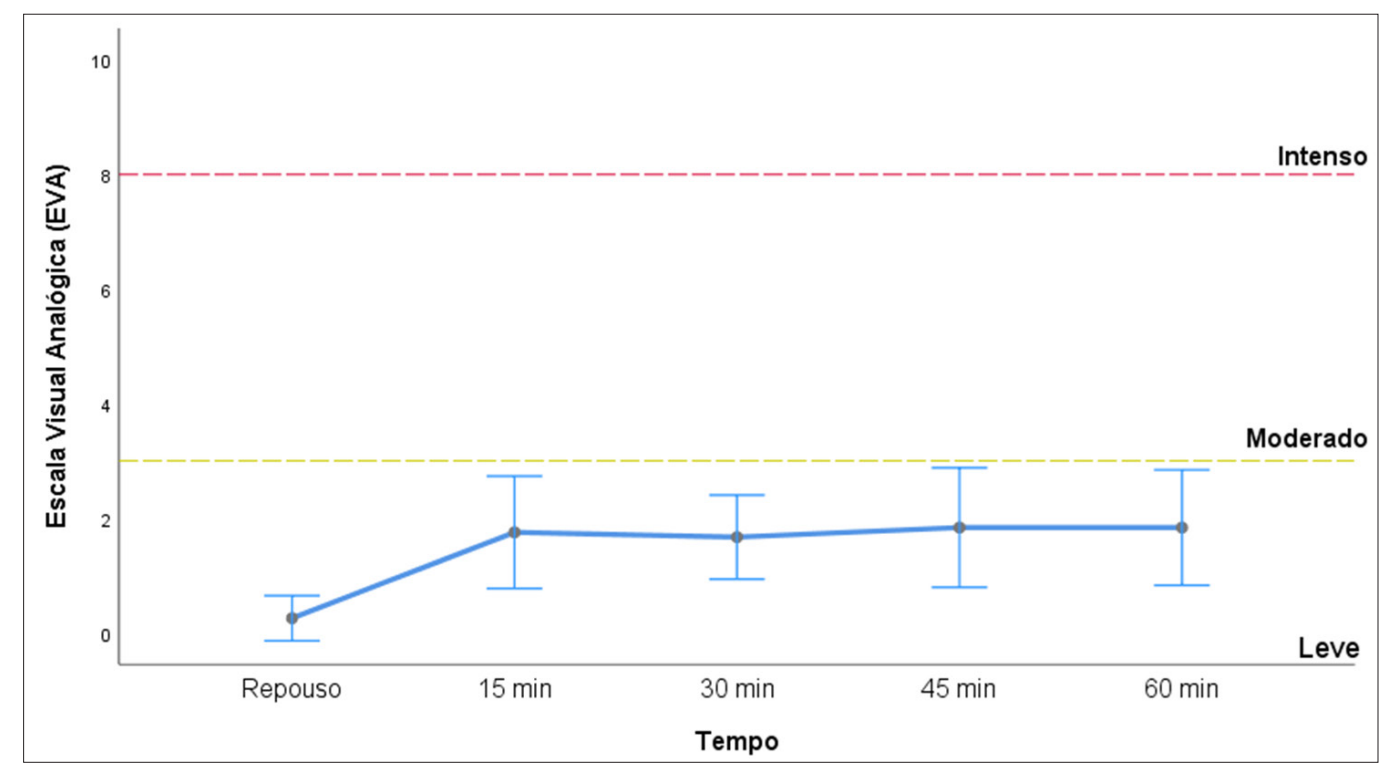

Figura 7. Efeitos adversos da interface avaliados pelos voluntários ao final do uso da interface.

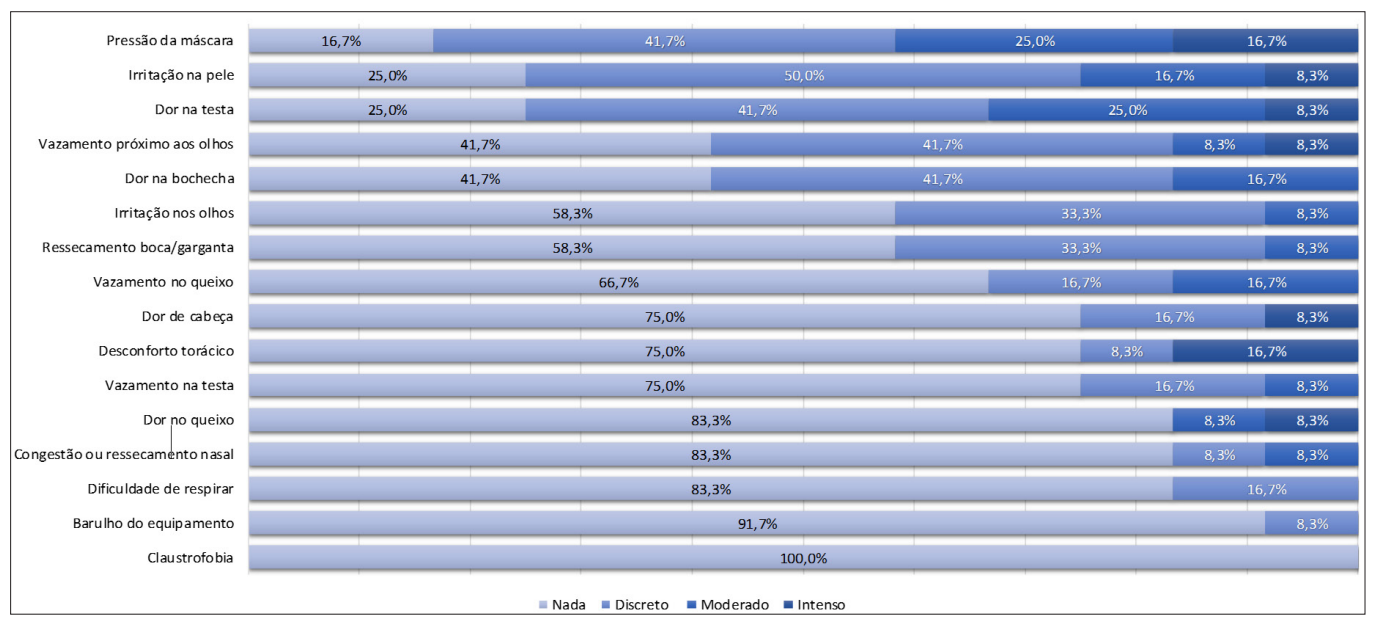




\section{DISCUSSÃO}

O presente trabalho é o primeiro do tipo realizado no Brasil que avaliou uma interface máscara de mergulho como estratégia de suporte ventilatório adaptada para a ventilação não invasiva, a saber: parâmetros fisiológicos agudos, nível de conforto e efeitos adversos.

Em estudo realizado por Bibiano-Guillen e colaboradores (2021), os autores avaliaram a eficácia de um protótipo de máscara de mergulho adaptada (máscaras de mergulho com snorkel modificadas) para suporte ventilatório não invasivo, em 25 pacientes com sintomas respiratórios agudos secundária à infecção por SARS-CoV-2 (infecção pelo novo coronavírus), e concluíram que o dispositivo apresentou boa tolerância e poucos efeitos adversos significativos, porém, a sua aplicação foi indicada no contexto da pandemia (COVID-19) e na profunda escassez de outras alternativas de suporte ventilatório não invasivo, sendo necessário um número maior de casos para apoiar e validar estatisticamente estes resultados. ${ }^{18}$ Em 2020, um relato de caso avaliou uma paciente com COVID-19 grave admitida em unidade de terapia intensiva (UTI) e submetida à VNI através de uma máscara de mergulho associada a posição prona, e demonstrou ser eficaz em evitar a intubação orotraqueal dessa paciente com COVID-19 grave, ressaltando a importância da intervenção proposta. ${ }^{19}$ No presente estudo, a máscara de mergulho também pareceu ser uma interface viável, não apresentando graves níveis de desconforto ou efeitos adversos que inviabilize a sua utilização, porém, para ampliar a sua aplicabilidade clínica, é importante a implementação de melhorias para melhor adaptação, funcionalidade e conforto do paciente.

No estudo Saatci et al., (2004), observou-se que a reinalação de $\mathrm{CO}_{2}$ foi um efeito adverso presente no uso da VNI, porém em menor proporção com uso da máscara facial total. Importante ressaltar ainda, que no estudo de Saatci (2004) haviam orifícios de exalação na máscara facial total, o que difere da máscara de mergulho. ${ }^{20}$ No presente estudo, para a análise da reinalação de $\mathrm{CO}_{2}$ foi realizado gasometria venosa de cada voluntário antes e após o uso da interface máscara de mergulho, e não foi observada diferença estatisticamente significante $(p=0,928)$.

Em estudo realizado por Holanda et al. (2009), esses autores analisaram os efeitos adversos no uso da VNI em três tipos diferentes de interfaces: máscara facial total, facial e nasal, e não observaram diferenças entre as interfaces quanto ao nível de conforto. ${ }^{9}$ Em nosso estudo o nível de desconforto foi pontuado no decorrer dos 60 minutos, através do uso da EVA, e durante todo o experimento os níveis de desconforto mantiveram-se em leves, não sendo observado nenhuma situação de desconforto moderado ou intenso com o uso da interface.

Na presente pesquisa a sensação de claustrofobia não foi um problema, concordando com o estudo de Roy et al (2007), onde os autores também encontraram menores incidências de claustrofobia com o uso da MFT. ${ }^{21}$ Porém, em estudo realizado por Holanda et al. (2009), os autores relataram a claustrofobia e o ressecamento oronasal como os efeitos adversos mais frequentes durante utilização da MFT. $^{9}$ Acreditamos que a manutenção do campo de visão favorável da interface pode ter atenuado a sensação claustrofóbica, e que nenhum dos voluntários do estudo era suscetível a esse efeito. $\mathrm{O}$ ressecamento nasal, oral e irritação nos olhos também não foram vistos como um problema pela maior parte da amostra, o que pode-se atribuir esse resultado ao uso de filtro aquecedor e umidificador de ar utilizado em nosso modelo. ${ }^{22}$

A presença de vazamento foi avaliada em três pontos: na testa, próximo aos olhos e no queixo. Apesar de alguns voluntários não relatarem o vazamento próximo a testa como um problema, foi necessária frequente adaptação da interface pelos profissionais da equipe por presença de vazamentos, principalmente na região temporal e abaixo da linha do zigomático. Porém, vale ressaltar que não foi possível mensurar o grau de vazamento, e este fator aumenta as chances de ressecamento oronasal e consequentemente, falha da $\mathrm{VNI} .^{23}$

Além dos vazamentos, a pressão de ar excessiva na face e dor facial também são frequentemente citados como problemas no uso de interfaces durante a VNI. ${ }^{15}$ Corroborando com esses dados, no presente estudo a pressão da máscara foi relatada como um problema intenso por alguns voluntários e outros consideraram um problema discreto. A dor facial foi analisada em diferentes pontos: testa, bochechas e queixo, sendo considerada, dor na testa e bochecha como um problema discreto, e a dor no queixo não foi considerada um problema.

Em nosso instrumento de coleta de dados haviam citados 16 efeitos adversos, e os voluntários foram incentivados a descrever ainda quaisquer outros efeitos adversos que fossem necessários. Dentre os outros efeitos adversos relatados estavam: peso da máscara e pressão no ouvido, descritos como um grande problema. Ao encontro do relatado, Gay (2009) descreveu em seu estudo que uma das complicações do uso da VNI foi a pressão ou fluxo de ar que pode gerar desconforto no ouvido. ${ }^{24}$ Com relação ao peso da máscara, não encontramos descrição na literatura relatando como efeito adverso ou queixa referida durante o uso da VNI. Em adicional, avaliamos ainda o barulho produzido pelo equipamento, porém, para a maioria dos voluntários avaliados não foi considerado um problema. Vale salientar também que alguns indivíduos do estudo (dois voluntários) podem ter apresentado baixa tolerabilidade ao método, pois interromperam o protocolo por bradicardia durante o uso da interface, e a instabilidade hemodinâmica é considerada uma contra-indicação para o uso da VNI, limitando consideravelmente o seu uso. ${ }^{25}$

\section{Limitações do estudo}

Uma limitação da máscara de mergulho é o vazamento, pois o modelo sugerido não dispõe de um equipamento específico para VNI, e, portanto, não há compensação e nem quantificação dos vazamentos, o que predispõe a falha da terapêutica. Esse vazamento pode ter sido favorecido pelo peso da máscara, que dificulta o manuseio e foi referido como um grande problema, e pode ter gerado a necessidade da intensificação no ajuste da máscara de mergulho, e dessa forma acarretado aumento do desconforto pela pressão em pontos da face. 
Outro fator que pode ter facilitado a presença de vazamentos é o tamanho da interface, pois o estudo contava apenas com um tamanho de interface para os diversos voluntários avaliados. E ainda, outro aspecto limitante foram as fixações de ajuste serem fixadas (não removíveis) à máscara de mergulho, pois modelos utilizados na prática clínica são utilizados clipes e fixações móveis para facilitar a instalação e adaptação do paciente à VNI, o que não foi o caso em nosso estudo. Além disso, a amostra do estudo foi composta apenas por mulheres, este foi um fato inesperado, acreditamos que a adesão a pesquisa foi maior pela população do sexo feminino, porém, esse resultado é um aspecto limitante e pode mascarar os resultados do estudo.

\section{Implicações clínicas e perspectivas}

A máscara de mergulho poderá ser mais um equipamento para contribuir na assistência aos pacientes com insuficiência respiratória aguda ou crônica. Podendo ser utilizada em ambientes que não dispõem de ventilador mecânico, pois funciona também conectado a geradores de fluxos de ar comprimido e oxigênio.

Entretanto, trata-se de uma interface adaptada sendo necessário atentar-se às principais queixas relatadas no presente estudo. Além disso, é importante também identificar suas limitações

\section{REFERÊNCIAS}

1. Peter JV, Moran JL, Phillips-Hughes J, Warn D. Noninvasive ventilation in acute respiratory failure - a meta-analysis update. Crit Care Med. 2002;30(3):555-62.

2. Duca A, Rosti V, Brambilla AM, Cosentini R. Non-invasive ventilation in COPD exacerbation: how and why. Intern Emerg Med. 2019;14(1):139-42.

3. Bello G, De Pascale G, Antonelli M. Noninvasive ventilation. Clin Chest Med. 2016;37(4):711-21.

4. Pinheiro BV. Thermography as a tool for monitoring the interface between the noninvasive ventilation mask and the skin. J Bras Pneumol. 2017;43(2):81-2.

5. Azevedo LC, Park M, Salluh JI, Rea-Neto A, Souza-Dantas VC, Varaschin P, et al. Clinical outcomes of patients requiring ventilatory support in Brazilian intensive care units: a multicenter, prospective, cohort study. Crit Care. 2013;17(2):R63.

6. Liu J, Duan J, Bai L, Zhou L. Noninvasive ventilation intolerance: characteristics, predictors, and outcomes. Respir Care. 2016;61(3):277-84.

7. Nava S, Ceriana P. Causes of failure of noninvasive mechanical ventilation. Respir Care. 2004;49(3):295-303.

8. Nakamura MA, Costa EL, Carvalho CR, Tucci MR. Performance of ICU ventilators during noninvasive ventilation with large leaks in a total face mask: a bench study. J Bras Pneumol. 2014;40(3):294-303.

9. Holanda MA, Reis RC, Winkeler GF, Fortaleza SC, Lima JW, Pereira ED. Influence of total face, facial and nasal masks on short- e necessidade de adaptações que favoreçam instalação, manuseio e conforto da interface.

\section{CONCLUSÃO}

Os efeitos adversos identificados no presente estudo relacionados ao uso da interface máscara de mergulho como suporte ventilatório não invasivo já são conhecidos na literatura, como: pressão da máscara, desconforto torácico, vazamento, dificuldade de respirar, dentre outros. Porém, vale ressaltar que se trata de uma interface adaptada que poderá contribuir no desenvolvimento de inovações de interfaces sendo necessário pontuar as principais queixas identificadas e resolvê-las, de preferência antes da finalização do dispositivo de inovação, bem como investigar a aplicabilidade deste sob a perspectiva de um profissional de saúde quanto à instalação e manuseio durante o uso, para que estudos futuros possam esclarecer sobre seus resultados em indivíduos com insuficiência respiratória.

Portanto, a máscara de mergulho parece ser uma interface viável, não apresentando graves níveis de desconforto ou efeitos adversos que inviabilizem a sua utilização, porém, para ampliar a sua aplicabilidade clínica são necessárias implementações de melhorias.

term adverse effects during noninvasive ventilation. J Bras Pneumol. 2009;35(2):164-73.

10. Hill NS. Complications of noninvasive positive pressure ventilation. Respir Care. 1997;42(4):432-42.

11. Pontes SM, Melo LH, Maia NP, Nogueira AN, Vasconcelos TB, Pereira ED, et al. Influence of the ventilatory mode on acute adverse effects and facial thermography after noninvasive ventilation. J Bras Pneumol. 2017;43(2):87-94.

12. Schettino GP, Chatmongkolchart S, Hess DR, Kacmarek RM. Position of exhalation port and mask design affect $\mathrm{CO} 2$ rebreathing during noninvasive positive pressure ventilation. Crit Care Med. 2003;31(8):2178-82.

13. Barbas CS, Isola AM, Farias AM, Cavalcanti AB, Gama AM, Duarte AC, et al. Brazilian recommendations of mechanical ventilation 2013. Part I. Rev Bras Ter Intensiva. 2014;26(2):89121.

14. Díaz GG, Alcaraz AC, Talavera JC, Pérez PJ, Rodriguez AE, Cordoba FG, et al. Noninvasive positive-pressure ventilation to treat hypercapnic coma secondary to respiratory failure. Chest. 2005;127(3):952-60.

15. Hoeven MV, Brouwer E, Blanco CE. Nasal high frequency ventilation in neonates with moderate respiratory insufficiency. Arch Dis Child. 1998;79:61-3.

16. Profili J, Dubois EL, Karakitsos D, Hof LA. Overview of the user experience for snorkeling mask designs during the COVID-19 pandemic. Healthcare (Basel). 2021;9(2):204. 
17. Mehta S, Hill NS. Noninvasive ventilation. Am J Respir Crit Care Med. 2001;163(2):540-77.

18. Bibiano-Guillen C, Arias-Arcos B, Collado-Escudero C, MirMontero M, Corella-Montoya F, Torres-Macho J, et al. Adapted diving mask (ADM) device as respiratory support with oxygen output during COVID-19 pandemic. Am J Emerg Med. 2021;39:427.

19. Wagner LE, Basegio KG, Dornelles CF, Foernges R, Gaedke MA, Silva AL, et al. Diving mask adapted for non-invasive ventilation and prone position in a patient with severe Covid-19: case report. J Epidemiol Infect Control. 2020;10(3):1-8.

20. Saatci E, Miller DM, Stell IM, Lee KC, Moxham J. Dynamic dead space in face masks used with noninvasive ventilators: a lung model study. Eur Respir J. 2004;23(1):129-35.
21. Roy B, Cordova FC, Travaline JM, D’Alonzo GE Júnior, Criner GJ. Full face mask for noninvasive positive-pressureventilation in patients with acute respiratory failure. J Am Osteopath Assoc. 2007;107(4):148-56.

22. Araújo MT, Vieira SB, Vasquez EC, Fleury B. Heated humidification or face mask to prevent upper airway dryness during continuous positive airway pressure therapy. Chest. 2000;117(1):142-7.

23. Crimi C, Noto A, Princi P, Esquinas A, Nava S. A European survey of noninvasive ventilation practices. Eur Respir J. 2010;36(2):362-9.

24. Gay PC. Complications of noninvasive ventilation in acute care. Respiratory Care. 2009;54(2):246-57.

25. Schettino GP, Reis MA, Galas F, Park M, Franca S, Okamoto V. Ventilação mecânica não invasiva com pressão positiva. J Bras Pneumol. 2007;33(Suppl 2): 92-105.

\section{Como citar:}

Nogueira AN, Sousa BG, Sinhorelli JG, Vasconcelos RS, Viana SM, Beltrão BA, et al. Efeitos adversos agudos e conforto da interface máscara de mergulho como estratégia de suporte ventilatório durante a ventilação não invasiva. Rev Med UFC. 2021;61(1):1-8. 